Global Journal of Pure and Applied Mathematics.

ISSN 0973-1768 Volume 12, Number 2 (2016), pp. 1743-1751

(C) Research India Publications

http://www.ripublication.com/gjpam.htm

\title{
Scheduling of tollbooth collectors in a Jakarta toll road section
}

\author{
Farida Hanum, Putri Putu Pratami, Toni Bakhtiar \\ Division of Operations Research, \\ Department of Mathematics \\ Bogor Agricultural University, \\ Dramaga, Bogor 16680, Indonesia.
}

\begin{abstract}
The present paper discussed a simple but specific case of scheduling tollbooth collectors in a toll section part of Jakarta toll road network. A number of requirements asked by toll road management were considered alongside with goal preferences in order to provide an efficient timetable for tollbooth collectors. A non-preemptive goal programming approach were employed to simultaneously minimized the shortage of workdays from its ideal level and the number of off-on-off scheduling patterns.
\end{abstract}

AMS subject classification: 90B35, 90B50, 90C29.

Keywords: Tollbooth collectors, scheduling, goal programming, Jakarta toll road.

\section{Introduction}

In 2013, the toll road network in Indonesia spans along 784 kilometers in four major islands: Jawa, Sumatra, Sulawesi and Bali. The network is expected to be expanded for another 534 kilometers in the near future with highest priority. However, more than 90 percent of the existing toll road system is operated in Jawa and half of that, which consists of 14 toll segments, connects several pivotal loci within greater area of capital city Jakarta. PT Jasa Marga is a big player of toll road management company as they administer 15 out of 29 toll segments in Indonesia [9].

In such company or any other organizations with large number of employees, staff scheduling is one of the most important factors of workforce management strategy and the one that is most prone to errors or issues. Scheduling the perfect workforce plan is 
quite a task. It is not only about scheduling the personnel with the right skills, but also about assigning the personnel while taking their preferences and company requirements into account $[1,8,10]$. For a toll road management, the scheduling of tollbooth collectors is an challenging problem as it may exhibit unique features.

Research by [3] might be the first exertion on the application of linear programming on toll collectors scheduling, where the working regulations for full-time and part-time shifts was considered and eight-phase algorithm was constructed. In [11], integer programming models were formulated to determine the number of toll booths required to satisfy vehicle demands and then the manpower shifts. And more recently, it was considered in [7] an integer linear programming problem of general daily staff scheduling problem with hourly requirement patterns illustrated in scheduling toll booth collectors of full-timers and part-timers. Another direction of research in this topic relating to the operational of tollbooth, such as design and operation policy [6], pricing [2], and queueing [4].

In the present paper we consider a specific tollbooth collectors scheduling problem facing in Jakarta toll road. Our works is motivated by fact that in the end of 2014 around 70 percent of tollbooths in Indonesia are still manned, i.e., the booth houses a worker who accepts the toll and can provide change and a receipt if needed, and the rests are already applying the electronic toll collecting (ETC) system. A non-preemptive goal programming approach were adopted to simultaneously minimized the shortage of workdays from its ideal level and the number of off-on-off scheduling patterns. It is wellknown that the goal programming method is commonly exploited in accommodating a number of preferences as did in [5]. The model is featured within the setting of toll collectors scheduling in a certain section of Jakarta toll road, namely Pelabuhan Section.

\section{Description of the scheduling problem}

Pelabuhan Section is a part of the Jakarta Intra-urban Toll Road jointly operated by PT Jasa Marga and PT Citra Marga Nusaphala Persada. This section, in fact managed by the later company, is 13 kilometers spanned from Pluit to Tanjung Priok Seaport. Seven toll gates are operated in this road section which consists of 21 booths in total (see Table 1) and all are in-flow type booths. To operate all the booths, the company employs 78 full-time workers, which consist of 26 woman-staffs and 52 man-staffs. The workforce scheduling is constructed with respect to the company requirements. Some of these necessities are the followings:

1. The work day is a full day long, i.e., 24 hours, which divided into three shifts: morning shift (05.30-13.30), afternoon shift (12.30-20.30) and night shift (19.3006.30). There is a 1-hour intersection between two shifts intended as a transition time.

2. Woman-staffs work only in morning shift, whereas man-staffs work either in afternoon or night shifts.

3. Every staff will get some day-offs during the period of scheduling. 
4. The number of staffs needed varies among shifts. It depends on the traffic volume of certain shift in certain day, which means that some booths may not be opened. It is assumed that the not-opened booths are known based on historical data.

5. A staff works only one shift per day, otherwise he/she has a day-off.

6. Other company policies for the shift assignment, such as a staff does not work in the same booth in two consecutive days and the assignment of a given staff to a specific shift cannot exceed predefined upper limit.

The aforementioned requirements alongside with other common conditions imposed in scheduling problem, e.g., uniqueness, will represent as hard constraints, a set of must be satisfied prerequisites. On the other hand, in order to respect some preferences we also consider a few number of soft constraints, a set of not necessary to be satisfied conditions which is then formulated as goals. We need to achieve these goals as close as possible. In this work we consider to achieve as small as possible the shortage of workdays from its ideal level and the number of off-on-off scheduling patterns.

\section{Mathematical model}

To formulate the optimization model, we firstly introduce the notion of sets and variables used throughout the paper. We denote by $\mathbb{S}_{0}=\{1, \ldots, 26\}$ and $\mathbb{S}_{1}=\{27, \ldots, 78\}$ sets of all woman-staffs and man-staffs, respectively. Thus by $\mathbb{S}:=\mathbb{S}_{0} \cup \mathbb{S}_{1}$ the set of all staffs. We introduce $\mathbb{D}=\{1, \ldots, T\}$ as the set of all days in the period. We denote by $\mathbb{B}_{m}, \mathbb{B}_{a}$ and $\mathbb{B}_{n}$ sets of all opened booths in morning $(m)$, afternoon $(a)$ and night (n) shifts, respectively. Based on historical record we have $\mathbb{B}_{m}=\{1, \ldots, 5,8, \ldots, 20\}$, $\mathbb{B}_{a}=\{1, \ldots, 20\}$ and $\mathbb{B}_{n}=\{1,2,3,6,8,9,12,14,15,16,17,19,20,21\}$. We refer to Table 1 for the name of booths.

Our decision variables are defined as follow:

$$
X_{i j k}^{m}= \begin{cases}1 ; & \text { if staff } i \text { works at booth } j \text { at morning shift of day } k \\ 0 & ; \quad \text { otherwise }\end{cases}
$$

Table 1: The tollbooth of Pelabuhan Section.

\begin{tabular}{clcl}
\hline No & Name of Gate & Number of Booths & Index of Booth \\
\hline 1 & Kemayoran & 5 & $1,2,3,4,5$ \\
2 & Pluit & 3 & $6,7,8$ \\
3 & Ancol Barat & 5 & $9,10,11,12,13$ \\
4 & Gedong Panjang I & 2 & 14,15 \\
5 & Gedong Panjang II & 2 & 16,17 \\
6 & Jembatan Tiga I & 2 & 18,19 \\
7 & Jembatan Tiga II & 2 & 20,21 \\
\hline
\end{tabular}




$$
\begin{aligned}
X_{i j k}^{a} & =\left\{\begin{array}{lll}
1 & ; & \text { if staff } i \text { works at booth } j \text { at afternoon shift of day } k \\
0 & ; & \text { otherwise, }
\end{array}\right. \\
X_{i j k}^{n} & =\left\{\begin{array}{lll}
1 & ; & \text { if staff } i \text { works at booth } j \text { at night shift of day } k \\
0 & ; & \text { otherwise, }
\end{array}\right. \\
Y_{i k} & =\left\{\begin{array}{lll}
1 & ; & \text { if staff } i \text { has day-off at day } k \\
0 & ; & \text { otherwise. }
\end{array}\right.
\end{aligned}
$$

\subsection{Hard constraints}

In this part we present all requirements must be satisfied by the model.

1. Woman-staffs are assigned only in the morning shift and man-staffs are not. Thus for all $k \in \mathbb{D}$ we have

$$
\sum_{i \in \mathbb{S}_{1}} \sum_{j \in \mathbb{B}_{m}} X_{i j k}^{m}=0, \quad \sum_{i \in \mathbb{S}_{0}} \sum_{j \in \mathbb{B}_{a}} X_{i j k}^{a}=0, \quad \sum_{i \in \mathbb{S}_{0}} \sum_{j \in \mathbb{B}_{n}} X_{i j k}^{n}=0 .
$$

2. There must be an adequate number of staffs available working in each shift every day. Thus for all $k \in \mathbb{D}$ we have

$$
\sum_{i \in \mathbb{S}_{0}} \sum_{j \in \mathbb{B}_{m}} X_{i j k}^{m} \geq \alpha_{k}^{m}, \quad \sum_{i \in \mathbb{S}_{1}} \sum_{j \in \mathbb{B}_{a}} X_{i j k}^{a} \geq \alpha_{k}^{a}, \quad \sum_{i \in \mathbb{S}_{1}} \sum_{j \in \mathbb{B}_{n}} X_{i j k}^{n} \geq \alpha_{k}^{n}
$$

Here we have $\alpha_{k}^{m}=18, \alpha_{k}^{a}=20$ and $\alpha_{k}^{n}=14$, the minimum number of staffs required in each shift for all days.

3. No staff is assigned to a booth which is unopened. Thus for all $i \in \mathbb{S}$ and for all $k \in \mathbb{D}$,

$$
X_{i j k}^{m}=0, \forall j \notin \mathbb{B}_{m} ; \quad X_{i j k}^{a}=0, \forall j \notin \mathbb{B}_{a} ; \quad X_{i j k}^{n}=0, \forall j \notin \mathbb{B}_{n} .
$$

4. Every staff should be assigned to at most one shift for each day, otherwise he/she gets a day-off:

$$
Y_{i k}+\sum_{j \in \mathbb{B}_{m}} X_{i j k}^{m}+\sum_{j \in \mathbb{B}_{a}} X_{i j k}^{a}+\sum_{j \in \mathbb{B}_{n}} X_{i j k}^{n}=1
$$

for all $i \in \mathbb{S}$ and for all $k \in \mathbb{D}$.

5. Every staff should be assigned to at most one booth in one shift. Accordingly, for all $k \in \mathbb{D}$ it is imposed that

$$
\sum_{j \in \mathbb{B}_{m}} X_{i j k}^{m} \leq 1, \forall i \in \mathbb{S}_{0} ; \quad \sum_{j \in \mathbb{B}_{a}} X_{i j k}^{a} \leq 1, \forall i \in \mathbb{S}_{1} ; \quad \sum_{j \in \mathbb{B}_{n}} X_{i j k}^{n} \leq 1, \forall i \in \mathbb{S}_{1}
$$


6. One booth can only house at most one staff. Therefore, for all $k \in \mathbb{D}$ it should be satisfied that

$$
\sum_{i \in \mathbb{S}_{0}} X_{i j k}^{m} \leq 1, \forall j \in \mathbb{B}_{m} ; \quad \sum_{i \in \mathbb{S}_{1}} X_{i j k}^{a} \leq 1, \forall j \in \mathbb{B}_{a} ; \quad \sum_{i \in \mathbb{S}_{1}} X_{i j k}^{n} \leq 1, \forall j \in \mathbb{B}_{n} .
$$

7. Within one period of scheduling, every staff cannot work more than $\beta$ consecutive days. In other word, he/she must have at least one day-off:

$$
Y_{i k}+Y_{i, k+1}+Y_{i, k+2}+\cdots+Y_{i, k+\beta} \geq 1,
$$

for all $i \in \mathbb{S}$ and $k=1,2, \ldots, T-\beta$. In this situation, company regulation gives $\beta=4$.

8. Regulation prevents staffs for working in the same shift more than certain upper bounds. For woman-staffs, since they work only in the morning, the bound means the maximum number of days on. For man-staff, this constraint will evenly distribute afternoon and night shifts.

$$
\begin{aligned}
& \sum_{k \in \mathbb{D}} \sum_{j \in \mathbb{B}_{m}} X_{i j k}^{m} \leq \gamma_{m}, \quad \forall i \in \mathbb{S}_{0}, \\
& \sum_{k \in \mathbb{D}} \sum_{j \in \mathbb{B}_{a}} X_{i j k}^{a} \leq \gamma_{a}, \quad \forall i \in \mathbb{S}_{1}, \\
& \sum_{k \in \mathbb{D}} \sum_{j \in \mathbb{B}_{n}} X_{i j k}^{n} \leq \gamma_{n}, \quad \forall i \in \mathbb{S}_{1},
\end{aligned}
$$

where $\gamma_{m}=22$ and $\gamma_{a}=\gamma_{n}=13$.

9. Since there are 21 booths in total, staffs should not be assigned more than certain times in the same booth within period:

$$
\begin{aligned}
& \sum_{k \in \mathbb{D}} X_{i j k}^{m} \leq r_{m}, \quad \forall i \in \mathbb{S}_{0}, \forall j \in \mathbb{B}_{m}, \\
& \sum_{k \in \mathbb{D}} X_{i j k}^{a} \leq r_{a}, \quad \forall i \in \mathbb{S}_{1}, \forall j \in \mathbb{B}_{a}, \\
& \sum_{k \in \mathbb{D}} X_{i j k}^{n} \leq r_{n}, \quad \forall i \in \mathbb{S}_{1}, \forall j \in \mathbb{B}_{n} .
\end{aligned}
$$

In fact, we set $r_{m}=r_{a}=r_{n}=2$.

\subsection{Goal constraints}

The goal programming model is probably the most known in mathematical programming to incorporate the decision-maker's preferences in term of multiple objectives. There are two preferences considered in this work, namely the less shortage of total workdays and the less number of off-on-off scheduling patterns. 
1. It is more preferable that staffs have the total number of days-on around their ideal level. Thus, if there is shortage, we want to keep the shortage minimum.

$$
\begin{aligned}
& \sum_{k \in \mathbb{D}} \sum_{j \in \mathbb{B}_{m}} X_{i j k}^{m}-a_{i}^{+}+a_{i}^{-}=\bar{a}, \quad \forall i \in \mathbb{S}_{0}, \\
& \sum_{k \in \mathbb{D}} \sum_{j \in \mathbb{B}_{a}} X_{i j k}^{a}-b_{i}^{+}+b_{i}^{-}=\bar{b}, \quad \forall i \in \mathbb{S}_{1}, \\
& \sum_{k \in \mathbb{D}} \sum_{j \in \mathbb{B}_{n}} X_{i j k}^{n}-c_{i}^{+}+c_{i}^{-}=\bar{c}, \quad \forall i \in \mathbb{S}_{1},
\end{aligned}
$$

where $a_{i}^{+}, b_{i}^{+}$and $c_{i}^{+}$denote the positive deviation from their ideal levels $\bar{a}, \bar{b}$ and $\bar{c}$, respectively. While $a_{i}^{-}, b_{i}^{-}$and $c_{i}^{-}$account the negative deviation. Here, $\bar{a}=21, \bar{b}=11$ and $\bar{c}=9$, which suggest that the expected workdays in one period is around 20 days.

2. Naturally, we prefer to have the fewest possible number of off-on-off working sequences. Thus, for all $k \in \mathbb{D}$ we have

$$
\begin{aligned}
Y_{i k}+\sum_{j \in \mathbb{B}_{m}} X_{i j, k+1}^{m}+Y_{i, k+2}-d_{i k}^{+}+d_{i k}^{-} & =2, \quad \forall i \in \mathbb{S}_{0}, \\
Y_{i k}+\sum_{j \in \mathbb{B}_{a}} X_{i j, k+1}^{a}+\sum_{j \in \mathbb{B}_{n}} X_{i j, k+1}^{n}+Y_{i, k+2}-e_{i k}^{+}+e_{i k}^{-} & =2, \quad \forall i \in \mathbb{S}_{1} .
\end{aligned}
$$

\subsection{Objective function}

Since we aim to minimized the shortage of workdays from its ideal level and at the same time the number of off-on-off scheduling patterns, then we employ a non-preemptive goal programming where all the goals are of roughly comparable importance. Thus, our objective function is

$$
\min _{X} z:=\sum_{i \in \mathbb{S}}\left(w_{a} a_{i}^{-}+w_{b} b_{i}^{-}+w_{c} c_{i}^{-}+\sum_{k \in \mathbb{D}}\left(w_{d} d_{i k}^{+}+w_{e} e_{i k}^{+}\right)\right),
$$

where $X=\left\{X_{i j k}^{m}, X_{i j k}^{a}, X_{i j k}^{n}, Y_{i k}\right\}$ is set of all decision variables. For the weights we set $w_{a}=4, w_{b}=w_{c}=3$ and $w_{d}=w_{e}=2$.

\section{Result and discussion}

Running of the program results the minimum objective function $z^{*}=102$, which contributed solely by $c_{i}^{-}$as other deviation variables are all zero. This means that by the schedule no one of woman-staffs will work in morning shift less than 21 times in one period, no one of man-staffs will work in afternoon shift less than 11 times, but some of 
Table 2: Summary of program output

\begin{tabular}{ccccc}
\hline Shift & Number of Duties & Number of Staffs & Percentage & Variance \\
\hline \multirow{2}{*}{ Morning } & 21 & 14 & 53.85 & \multirow{2}{*}{0.25} \\
& 22 & 12 & 46.15 & \\
\multirow{3}{*}{ Afternoon } & 11 & 23 & 44.23 & \\
& 12 & 10 & 19.23 & 0.80 \\
& 13 & 19 & 36.54 & \\
\multirow{5}{*}{ Night } & 5 & 2 & 3.85 & \\
& 6 & 4 & 7.69 & \\
& 7 & 2 & 3.85 & 1.24 \\
& 8 & 10 & 19.23 & \\
\multirow{5}{*}{ Day-off } & 9 & 25 & 65.38 & \\
& 1 & 22 & 32.05 & \\
& 11 & 17 & 28.21 & \\
& 12 & 9 & 21.79 & 1.57 \\
& 13 & 4 & 11.54 & \\
& 14 & 1 & 5.13 & \\
\hline
\end{tabular}

them will be on duties in night shift less than its ideal level, 9 times. Moreover, no one of staffs will have off-on-off working sequence.

Summary in Table 2 informs that the morning shifts are almost evenly distributed among 26 woman-staffs, where 14 staffs (54 percent) will work 22 times during the period, and the rests 21 times. The ideal level of working in the afternoon shift is 11 times. However, the number of duties in this shift varies among staffs. Most staffs (44 percent) will work in their ideal level, but some of them will have more than that, i.e., 12 times (19 percent) and 13 times ( 37 percent). Workload in night shifts is less evenly allocated. Even though most of staffs (65 percent) will enjoy their ideal level 9 times within period, nevertheless the rest of staffs will experience 5-8 times working in the night shift. Obviously, from the viewpoint of fairness, the schedule may not fulfill expectation some of staffs as they get more duties in the night, which are probably undesirable. Variation in workload subsequently affects that of day-off as shown in last rows.

\section{Concluding remark}

It has been shown that non-preemptive goal programming can be considered as a framework for modeling scheduling problem of tollbooth collectors. Among conflicting requirements, few of them are relaxed to become goals, which should as closely as possible be achieved. This strategy, however, increases the feasibility of the problem.

To improve the fairness, instead of minimizing the deviation from ideal level some may replace the objective function with one of minimizing the total variance $\sigma$ of the 
workload. For instance, we may try the following:

$$
\min \sigma:=\sum_{\ell=1}^{4} w_{\ell}\left[\frac{1}{N_{X}} \sum_{\ell \in \mathbb{S}_{X}} X_{\ell}^{2}-\frac{1}{N_{X}^{2}}\left(\sum_{\ell \in \mathbb{S}_{X}} X_{\ell}\right)^{2}\right],
$$

where $X_{\ell} \in\left\{X_{i j k}^{m}, X_{i j k}^{a}, X_{i j k}^{n}\right\}, \mathbb{S}_{X} \in\left\{\mathbb{S}_{0}, \mathbb{S}_{1}\right\}$ is the set of respecting staffs whose cardinality is $N_{X}$ and $w_{\ell}$ are the weights. Unfortunately this will lead to a nonlinear optimization, which is in general a harder problem.

\section{References}

[1] A. Aman, T. Bakhtiar, F. Hanum and P.T. Supriyo, "OR/MS applications in Mt. Merapi disaster management," Journal of Mathematics and Statistics, 8(2): 264 273, 2012, doi: 10.3844/jmssp.2012.264.273.

[2] L. Bai, D.W. Hearn and S. Lawphongpanich, "A heuristic method for the minimum toll booth problem," Journal of Global Optimization, 48(4): 533-548, 2010, doi: 10.1007/s10898-010-9527-7.

[3] J.L. Byrne and R.B. Potts, "Scheduling of toll collectors," Transportation Science, 7(3): 224-245, 1973, doi: 10.1287/trsc.7.3.224.

[4] T.V. Do, "A closed-form solution for a tollbooth tandem queue with two heterogeneous servers and exponential service times," European Journal of Operational Research, 247(2): 672-675, 2015, doi: 10.1016/j.ejor.2015.05.044.

[5] F. Hanum, M.A. Romliyah and T. Bakhtiar, "Exam invigilators assignment problem: a goal programming approach," Applied Mathematical Sciences, 9(58): 28712880, 2015, doi: 10.12988/ams.2015.52134.

[6] L.H. He, S.B. Li, Q. Gao and Z.G. Zhang, "Improving operational efficiency for a two-way highway toll station," Quality Technology \& Quantitative Management, 12(3): 353-366, 2015, doi: 10.1080/16843703.2015.11673386.

[7] N.H. Mohamad and F. Said, "Integer linear programming approach to scheduling toll booth collectors problem," Indian Journal of Science and Technology, 6(5): 4416-4421, 2013, doi: 10.17485/ijst/2013/v6i5/33241.

[8] S. Mohan, "Scheduling part-time personnel with availability restrictions and preferences to maximize employee satisfaction," Mathematical and Computer Modelling, 48: 1806-1813, 2007, doi: 10.1016/j.mcm.2007.12.027.

[9] P.U. Pusdata, 2013, Statistic of Public Work 2013, Jakarta: Ministry of Public Work of Indonesia. 
[10] M. Rocha, J.F. Oliveira and M.A. Carravilla, "Quantitative approaches on staff scheduling and rostering in hospitality management: an overview," American Journal of Operations Research, 2: 137-145, 2012, doi: 10.4236/ajor.2012.21016.

[11] J. Vittawasakul and J. Pichitlamken, "Planning of work schedules for toll booth collectors," Lecture Notes in Management Science, 4: 116-124, 2012. 